\title{
Online OSCE in pharmacy education: Strategies to preserve academic integrity of high-stakes assessment
}

\author{
Sunanthiny Krishnan ${ }^{1}$ (D), Sara Chuang ${ }^{2}$, Vivienne Mak $^{2}$ (D) \\ ${ }^{1}$ School of Pharmacy, Monash University Malaysia, Bandar Sunway, Selangor, Malaysia \\ ${ }^{2}$ Faculty of Pharmacy and Pharmaceutical Sciences, Monash University, Parkville, Australia
}

\section{Keywords}

Academic integrity

Cheating

Collusion

Online OSCE

Pharmacy education

\section{Correspondence}

Sunanthiny Krishnan

Monash University Malaysia

Bandar Sunway

Selangor 47500

Malaysia

sunanthiny.s.krishnan@monash.edu

\begin{abstract}
The Objective Structured Clinical Examination (OSCE) is a highly valued performancebased competency assessment that is extensively employed in medical and health professions education. In pharmacy undergraduate programmes, OSCE is an integral component of the curriculum, constituting both formative and summative assessments of the course. When the COVID-19 pandemic posed an overarching challenge in the delivery of face-to-face teaching and learning activities, academic institutions around the world ineluctably transitioned to online mode of education. Conducting OSCEs on virtual platforms presents its unique set of challenges. In the absence of physical isolation and invigilation of students, the risk of cheating and collusion is particularly high during virtual OSCEs. With the experience of conducting high-stakes OSCEs on virtual platforms at two different campuses simultaneously, the authors outline several strategies that can be implemented to ensure the academic integrity of the assessment.
\end{abstract}

\section{Introduction}

First introduced in 1975 (Harden et al., 1975), the Objective Structured Clinical Examination (OSCE) is an integral component of clinical assessments in medical and health professions education (Patricio et al., 2013; Shirwaikar, 2015; Prettyman et al., 2018; Hopwood et al., 2021). The OSCEs are considered the gold standard for assessing students' clinical competence due to their ability to simulate real-life scenarios. They are often used to assess students' competence in managing medical and/or medication-related problems with an equally weighted emphasis on interpersonal and communication skills in a controlled healthcare-like setting (Shirwaikar, 2015; Hopwood et al., 2021).

In many pharmacy undergraduate programmes, OSCEs are traditionally used as summative assessments to evaluate final-year students' preparedness for practice (Corbo et al., 2006; Al-Haqan et al., 2021). Nevertheless, many institutions find it useful to incorporate OSCEs at earlier stages of the course, as early as the first year of the programme (Mak et al., 2020; Al-Haqan et al., 2021; Savage et al., 2021).
At Monash University, OSCEs are given substantial emphasis in the curriculum of the pharmacy degree programme, forming a significant component of assessment throughout the course starting from year one up to year four. For final-year students, the exit OSCE is a hurdle requirement for graduation. The Monash pharmacy programme is delivered at two campuses concurrently, i.e., in Australia and Malaysia. The contents and assessments of the programme are similar at both campuses. As all examinations, including OSCEs, are conducted simultaneously at both the Australian and Malaysian campuses, robust coordination and thorough communication have been essential to safeguard the academic integrity of the assessment across both campuses.

A standard face-to-face OSCE set-up includes several examination booths, also known as "stations". These stations are either interactive or non-interactive (Shirwaikar, 2015). In the interactive stations, a simulated patient will role-play a scenario with the student. A trained examiner observes the interaction and assesses the student's performance. The non- 
interactive station is where the student will complete the assessment in a written exam format. There is no simulated patient in the non-interactive stations. Students will move from one station to another, solving problems at each station within a specified time limit until they complete a circuit consisting of a series of stations (Shirwaikar, 2015; Kristina et al., 2018).

Often, while a group of students are rotating through the OSCE circuit, the remaining students are held in a specified location, either before or after the examination, to reduce any risk of collusion between those who have completed the OSCE and those who are waiting to undertake the exam. Mobile phones and other forms of electronic devices are not permitted in the holding rooms to ensure that there is no communication between students during the examination period (Brown, 2015; Noonan et al., 2020). For Monash pharmacy OSCEs, this physical isolation also serves to prevent any cross-campus collusion between the Australian and Malaysian students.

In 2020, when the COVID-19 global pandemic forced nationwide lockdowns and closure of academic institutions, educators had to instantly adapt to the online mode of delivery to ensure continuity of teaching and assessment activities. Although online written assessments were not a novel concept in many higher academic institutions at the time, performancebased assessment, such as an OSCE on a virtual platform, was new, particularly in pharmacy programmes. Faculties were not only challenged with the logistical complexity of organising and coordinating an OSCE online, but the preservation of the academic integrity with this form of online assessment also required meticulous attention, especially in high-stakes OSCE.

The increasingly innovative communication methods, coupled with the absence of a physical holding area to invigilate students, increase the likelihood of collusion and cheating among students during online OSCEs. Students who undertake the OSCE later in the examination schedule may have an unfair advantage due to the potential sharing of examination information by those who have already completed the OSCE (Gotzmann et al., 2017; Ghouri et al., 2018; Noonan et al., 2020). Although anecdotal evidence shows that inadvertent exposure of examination materials to students prior to the OSCE (Wilkinson et al., 2003) or collusion among students during OSCE (Parks et al., 2006) does not always translate to higher marks in the 'advantaged' group of students, such breaches of academic integrity undeniably undermine the quality of the assessment.

Given the clandestine nature of academic misconduct, it is difficult to precisely identify points at which students will engage in collusion and/or cheating during online OSCEs. As such, careful planning of the examination with multiple check posts at various stages of the entire process is warranted to mitigate the risk of collusion and/or cheating.

With the experience of conducting high-stakes OSCEs on a virtual platform since 2020, the authors outline several strategies that can be implemented before, during and after an online OSCE to ensure the academic integrity of the assessment.

\section{Before online OSCE}

\section{Students to sign a Confidentiality Agreement Form}

Honesty and integrity are recognised as the core tenets of professionalism for pharmacy students (ACCP, 2009). Inculcating and reinforcing these values in the course of their study is essential in grooming future pharmacists who would be able to uphold solid ethical conduct and high standards of integrity that are germane to this profession.

Confidentiality Agreement Forms, also known as Academic Integrity Statements, are clauses that delineate the code of conduct expected of students while undertaking an assessment. Reviewing and signing this document prior to an online OSCE indirectly instils the notion of professional integrity among these students who are essentially pharmacists in training. It also effectively serves as a reminder of the institutional policies of academic integrity and the severe repercussions of any violation thereof, thus serving as a deterrent for students against breaching integrity.

The statements in the Agreement Form must be clearly phrased in a manner that expressly articulates the dishonest behaviour proscribed in the context of the online OSCE. This will add clarity to the document and establishe transparency in terms of the examination rules.

Examples of statements that can be included in the agreement:

- I will not seek assistance from or give assistance to anyone while undertaking the OSCE.

- I will not share any substance or content of the OSCE with anyone during or after the exam.

- I will not post or discuss any information regarding the OSCE on social media during or after the exam.

- I will not use any resources other than those permitted by the faculty or specified in the assessment information. 
Usage of generic blanket statements such as 'I pledge to abide by the University's academic integrity policies' may not serve its intended purpose as students often tend to acknowledge these unmindfully by means of ticking off the checkbox.

The Confidentiality Agreement Form can also be supplemented with a pre-OSCE quiz, which comprises questions pertaining to the academic integrity with regard to online OSCE and students' responsibilities. Students will need to determine if the questions/ scenarios presented to them constitute cheating and/or collusion and state their appropriate actions in these instances. To further emphasise the gravity of academic breaching, this quiz is made compulsory, and students must obtain a hundred per cent pass mark to be permitted to undertake the OSCE.

\section{Using a variety of OSCE case scenarios}

Using a variety of OSCE case scenarios is unquestionably a labour-intensive process but, by and large, is the only fool-proof method to curb collusion with the online OSCE. With each student assessed on a unique case scenario for the OSCE, the incentive for collusion is annihilated. Indeed, the very attempt of colluding could be counterproductive as the students are caught off guard with a new case scenario while they are preoccupied with details of a different case. Parks and collaborators (2006) described an incident of collusion among third-year medical students undertaking a pathology OSCE station whereby the histology picture was changed from tuberculosis on day 1 of the exams to squamous carcinoma on the second day. The rest of the case scenario, questions and marking rubric remained unchanged. The authors reported that a high number of candidates provided an incorrect diagnosis of tuberculosis on the second day of the exam, supporting the notion that collusion could disadvantage students.

The exercise of expanding the pool of exam questions by building a variety of case scenarios is prudently balanced against the sustainability of the process. In resourcelimited settings, it is still possible to achieve the ideal "one case per student" quotient by creating multiple variations of the core exam case scenario, thus generating several unique cases. These variations in the case details must be clinically relevant and significantly alter the final outcome of the cases, which subsequently minimises the collusion gain, if at all attempted.

When building different OSCE case scenarios, it is particularly useful to work within a defined framework of analytical checklist to maintain consistency of standards across these cases. Depending on the year level and difficulty, it is also worth aiming for exam cases that require higher-order thinking in Bloom's taxonomy (Bloom et al., 1956), rather than recall or comprehension questions, as the latter are generally less immune to cheating either individually or collectively as a group. Once the exam cases are built, a thorough review process, often requiring several reiterations, is called for to ensure the case scenarios are similar in terms of quality and difficulty so that no one student is advantaged over the other.

\section{Publishing exam schedules using student identification (ID) numbers only}

An individual student ID number is unique and private and is not readily accessible by other students. It is an institutional policy at Monash University that all schedules/timetables made available to students contain either student names or ID numbers but not both together. This policy is intended to protect students' privacy and to mitigate any wrongful identification and misuse of confidential information by any unauthorised parties.

For OSCE schedules, student ID numbers are used instead of names as the latter leads to direct recognition of members in the same exam time slots, which may increase the probability of collusion within the group.

\section{Releasing exam schedules closer to the examination day and time}

The premise behind this strategy is that when OSCE schedules are released very close to the examination day and time, it leaves little room for students to forge alliances with others to collude. This process works best when used in conjunction with releasing exam schedules using student ID numbers only.

General information about the exam times are released earlier to students, but the announcement of individual time slots for each student is reserved to the day before or in some cases on the day of the exam, with prior notice on the release date and time. The staggered release of timetables for students who will be sitting for the exam later in the day is adopted to maintain equity across the entire cohort.

With the exception of exam schedules, students must be adequately briefed and well informed about all other aspects of the virtual OSCE ahead of time, especially on the flow of the OSCE online to ensure smooth execution on the day.

\section{Setting a narrow examination window}

This strategy is particularly applicable for the noninteractive written OSCE stations that are undertaken online. These written stations are built onto the institution's Learning Management System (LMS), similar to any online assessment format. However, 
unlike other examinations, the written OSCE stations are time-pressed with tasks limited to under ten minutes (Shirwaikar, 2015). As such, aside from enabling the exact time limit for the attempt (i.e. the time the student starts and completes the task), the window period within which the examination remains available to students should also be restricted, as excessively wide opening time increases the risk of collusion (Figure 1). As a rule of thumb, if the OSCE station is timed ten minutes, a testing window of fifteen minutes is deemed sufficient, factoring in possible technical glitches of online assessments (Figure 2).

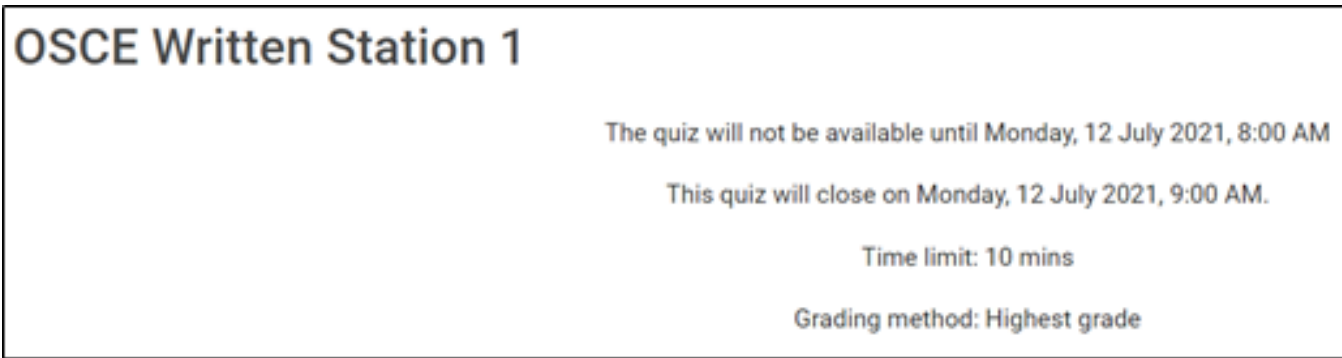

Figure 1: Time limit set-up (undesirable)

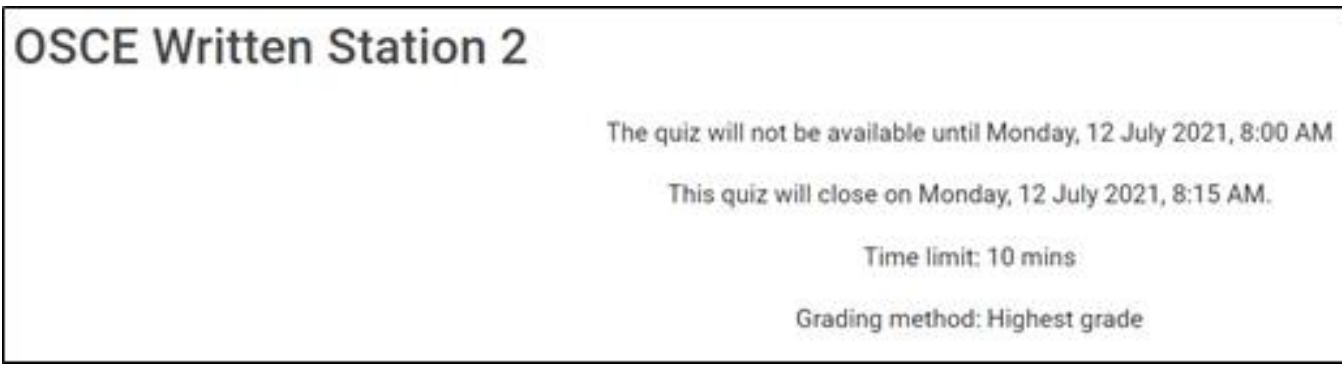

Figure 2: Time limit set-up (preferred)

As written OSCE stations are logistically challenging to be incorporated into the online circuit of active stations, the former is conducted separately (but within the OSCE day) with the entire cohort of students attempting the examination simultaneously. As such, with the examination set to local date and time on the LMS, it is crucial to clearly communicate the timings with students living in different time zones.

\section{During online OSCE}

\section{Using registered log-in to the online platform}

At Monash University, online OSCEs are undertaken on the Zoom platform, where OSCE administrators can restrict their examination to authenticated users only (Zoom, 2021). With such a set-up, anyone trying to log into the online OSCE using unregistered accounts will be denied access (Figure 3).

This setting can be restricted further by allowing only users with the university domain (i.e., university student email accounts), which deters others from sitting the OSCE in place of the original student. Authorising only authenticated users also allows for participant lists to be generated through Zoom, providing administrators with the list of students who attempted the online OSCE and the duration of attendance. Whilst the student's display name may be changed, the participation data is tied to the account. Therefore, an accurate record of participation can be maintained.

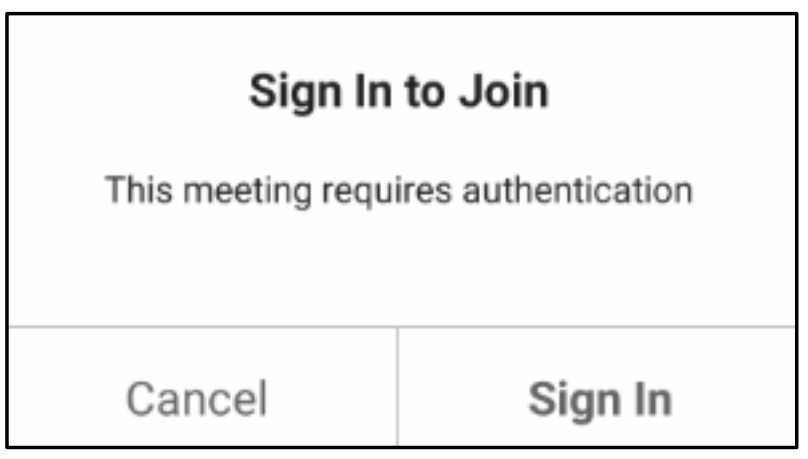

Figure 3: Sign-in requiring authentication 


\section{Requiring photo identification}

Impersonation in online examinations is a serious threat to the integrity of the assessment, and it occurs when an individual other than the student sits for the examination on behalf (Sheela, 2020), which generally transpires as the student believes that the impersonator, often a brighter student, would have a higher chance of passing the examination (Odero \& Oloko, 2013). In an institution with a large number of students undertaking the OSCE, it can be difficult to remember or recognise all the students. To circumvent the possibility of imposters, a photo ID is required for the online OSCE. Students are requested to present their student ID cards to the examiner prior to the start of the examination at their respective stations. The photo on the student ID card is cross-matched with the visuals of the student in tandem with their full name and student ID number to ensure that the individual in attendance is, in fact, the authentic candidate. Once this has been verified, they can then commence the OSCE.

\section{Imposing camera and audio requirements}

Students are mandated to keep their cameras and microphones switched on at all times during the OSCE to minimise any occurrence of students receiving assistance from external parties and to ensure that they are completing their online assessments individually. Similar to online invigilation, this allows examiners to be aware of all the verbal and visual cues at the students' end, such as unauthorised notes, signage or props, including any conversations students may have with anyone other than the simulated patient and/or message alerts on mobile phones and electronic devices. The use of cameras and microphones is a requirement if the institution is using online proctoring software.

\section{Sharing computer screen during online OSCE}

During online OSCE, students are required to display their computer desktops using the share screen function on the Zoom platform. Viewing students' navigation patterns on their computers, the type of resources used, and their approach to solving the OSCE cases enable examiners to gauge if students are working through the problem independently or if there is any suspicious activity. It also prevents students from using the device for any other purpose than for OSCE vis-a-vis communication or collusion with others. Aside from the deterrent aspect, this approach, which is similar to the think-aloud methodology (Mallinder \& Martini, 2021), also offers a greater insight into the students' thought process in terms of clinical decisionmaking, which may be helpful when providing feedback to students on their performance in the examination.

\section{Recording the online OSCE}

Students' performance during the online OSCE, in both active and written stations, are recorded for post-OSCE review. Aside from serving as evidence of attendance, these recordings are specifically valuable for the retrospective evaluation of cases that examiners flag up for suspicions of academic dishonesty. Similarly, these recordings can also be used for the purpose of proctor reviewing, whereby a trained proctor reviews them and highlights any questionable incidences for the faculty's consideration.

From a pedagogical perspective, the recordings of students' performance allow examiners to share detailed and personalised feedback with the students post-OSCE. Students may also review their own OSCE role-plays and reflect on how they could further improve their performance in formative assessment.

\section{Using online proctoring software}

Another way to tighten the grip on academic misconduct for written OSCEs is to employ online proctoring software. Examples of software include Examsoft (2021), ProctorU (2021) and Examity (2021). These programmes use artificial intelligence to analyse students' movements and their surroundings to detect any transgressive activities by accessing their webcams, computer screens, and microphones (Kharbat \& Abu Daabes, 2021; Li et al., 2021). However, the cost of eproctoring software can be prohibitive (Li et al., 2021).

\section{After online OSCE Delaying feedback}

OSCEs are often conducted over a long period of time (e.g. several days) due to the size of the student cohort. All feedback notes regarding the examination must be withheld until the entire cohort has completed the online OSCE to prevent an unfair advantage to any students. Examiners particularly are informed to refrain from discussing any individual student's performance while the OSCEs are still ongoing. In an incident of some students receiving advance information about an OSCE content as reported by Parks and colleagues (2006), those who did not receive the information understandably expressed feelings of inequity and a sense of devaluation of the assessment process. Therefore, academics and examiners should engage 
cautiously in any discussions related to the OSCE questions until the entire examination is completed.

\section{Conclusions}

As the new wave of virtual learning environments gathers momentum in the backdrop of the COVID-19 global pandemic, a paradigm shift in education is imminent. Extrapolating the experience from the pandemic, online OSCEs may offer a viable solution for competency assessments of offshore students who have the infrastructure to be assessed remotely. With telehealth becoming an increasingly popular trend in healthcare services, online OSCEs may also serve as a platform to assess skills to this end. Given the potential expansion of the value of online OSCEs, it is imperative that faculties undertake all the necessary steps to regulate the academic integrity to ensure this medium of assessment remains rigorous and relevant, especially for high-stakes OSCEs.

\section{Acknowledgements}

The authors would like to acknowledge the team of academics and teaching associates at Monash University Malaysia and Australia for their unwavering support for the smooth implementation of online OSCEs.

\section{References}

Al-Haqan, A., Al-Taweel, D., Koshy, S., \& Alghanem, S. (2021). Evolving to objective structured clinical exams (OSCE): transitional experience in an undergraduate pharmacy program in Kuwait. Saudi Pharmaceutical Journal, 29(1):104 -113. https://doi.org/10.1016/j.jsps.2020.12.013

American College of Clinical Pharmacy (ACCP), Holt, S. L., Lau, M. S., Wong, F. L., Attridge, R. T., Ho, M., Morris, J. L., Rutledge, E. D., Sapko, M. M., Tu, X., \& Vlasimsky, T. B. (2009). Tenets of professionalism for pharmacy students. Pharmacotherapy, 29(6), 757-759.

https://doi.org/10.1592/phco.29.6.757

Bloom, B.S., Engelhart, M.D., Furst, E.J., Hill, W.H., \& Krathwohl, D.R. (1956). Taxonomy of educational objectives: the classification of educational goals. Handbook 1: Cognitive domain. Longmans Green

Brown, C. (2015). Should we be "quarantining" students for objective structured clinical examinations (OSCEs)? Medical Teacher, 37(1):98-99.

https://doi.org/10.3109/0142159X.2014.940879

Corbo, M., Patel, J.P., Abdel Tawab, R., \& Davies, J.G. (2006). Evaluating clinical skills of undergraduate pharmacy students using objective structured clinical examinations (OSCEs). Pharmacy Education, 6(1):53-58

Examity (2021). Online proctoring on your terms. https://www.examity.com

ExamSoft (2021). Strengthen exam integrity with digital monitoring. http://examsoft.com/solutions/exam-monitor

Ghouri, A., Boachie, C., McDowall, S., Parle, J., Ditchfield, C.A., McConnachie, A., Walters, M.R., \& Ghouri, N. (2018). Gaining an advantage by sitting an OSCE after your peers: a retrospective study. Medical Teacher, 40(11):1136-1142. https://doi.org/10.1080/0142159X.2018.1458085

Gotzmann, A., De Champlain, A., Homayra, F., Fotheringham, A., De Vries, I., Forgie, M., \& Pugh, D. (2017). Cheating in OSCEs: the impact of simulated security breaches on OSCE performance. Teaching and Learning in Medicine, 29(1):52-58.

https://doi.org/10.1080/10401334.2016.1202832

Harden, R.M., Stevenson, M., Downie, W.W., \& Wilson, G.M. (1975). Assessment of clinical competence using objective structured examination. British Medical Journal, 1(5955):447-451. http://doi.org/10.1136/bmj.1.5955.447

Hopwood, J., Myers, G., Sturrock, A. (2021). Twelve tips for conducting a virtual OSCE. Medical Teacher, 43(6):633-636. http://doi.org/10.1080/0142159X.2020.1830961

Kharbat, F.F., Abu Daabes, A.S. (2021). E-proctored exams during the COVID-19 pandemic: a close understanding. Education and Information Technologies, 1-17. http://doi.org/10.1007/s10639-021-10458-7

Kristina, S.A., Gustriawanto, N., Rokhman, M.R., Aditama, H., \& Sari, I.P. (2018). Students' first experience with objective structured clinical examination in a pharmacy school in Indonesia. Journal of Applied Pharmaceutical Science, 8(9):102-106.

http://doi.org/10.7324/JAPS.2018.8915

Li, M., Luo, L., Sikdar, S., Nizam, N.I., Gao, S., Shan, H., Kruger, M., Kruger, U., Mohamed, H., Xia, L., \& Wang, G. (2021). Optimized collusion prevention for online exams during social distancing. NPJ Science of Learning, 6:5. http://doi.org/10.1038/s41539-020-00083-3

Mak, V., Sandhu, A.K., \& Krishnan, S. (2020). Using simulation to teach methods for improving patient literacy about medicines. Pharmacy, 8(4):192.

http://doi.org/10.3390/pharmacy8040192

Mallinder, A., \& Martini, N. (2021). Exploring community pharmacists' clinical decision-making using think aloud and protocol analysis. Research in Social and Administrative Pharmacy. http://doi.org/10.1016/j.sapharm.2021.05.002

Noonan, Z., Boyle, J.G., Pope, L., Ditchfield, C., Leach, J.P., \& McDowall, S. (2020). Quarantining the OSCE: reflections on the Glasgow experience. Clinical Teacher, 17: 94-97. http://doi.org/10.1111/tct.12993

Odero, T.A., \& Oloko, M.A. (2013). Effect of online registration on exam performance in Kenya Certificate of Secondary Education enrolment. A case of Sotik District, Kenya. American International Journal of Contemporary Research, 3(7):117-127 
Parks, R., Warren, P., Boyd, K., Cameron, H., Cumming, A., \& Lloyd-Jones, G. (2006). The objective structured clinical examination and student collusion: marks do not tell the whole truth. Journal of Medical Ethics, 32(12):734-738. http://doi.org/10.1136/jme.2005.015446

Patrício, M.F., Julião, M., Fareleira, F., \& Carneiro, A.V. (2013). Is the OSCE a feasible tool to assess competencies in undergraduate medical education? Medical Teacher, 35(6):503-514.

http://doi.org/10.3109/0142159X.2013.774330

Prettyman, A.V., Knight, E.P., \& Allison, T.E. (2018).

Objective structured clinical examination from virtually anywhere. Journal for Nurse Practitioners, 14(8): e157e163. http://doi.org/10.1016/j.nurpra.2018.05.007

ProctorU (2021). The leading proctoring solution for online exams. http://www.proctoru.com

Savage, A., Minshew, L.M., Anksorus, H.N., \& McLaughlin, J.E. (2021). Remote OSCE experience: what first year pharmacy students liked, learned, and suggested for future implementations. Pharmacy, 9(1):62.

http://doi.org/10.3390/pharmacy9010062

Sheela, S., Vanitha, V., Anne Ramya, T., \& Sobana, B. (2020). Detection of impersonation in online examination. Journal of Critical Reviews, 7(10): 2103-2109.

http://doi.org/10.31838/jcr.07.10.365

Shirwaikar, A. (2015). Objective structured clinical examination (OSCE) in pharmacy education - a trend. Pharmacy Practice, 13(4):627.

https://doi.org/10.18549/PharmPract.2015.04.627

Wilkinson, T., Fontaine, S., \& Egan, T. (2003). Was breach of examination security unfair in an objective structured clinical examination? A critical incident. Medical Teacher, 25(1):42-46.

http://doi.org/10.1080/0142159021000061413

Zoom (2021). Authentication Profiles for meetings and webinars. https://support.zoom.us/hc/enus/articles/360037117472-Authentication-Profiles-formeetings-and-webinars2021 